\title{
Sampling criteria and recording quality influence relaxation responses to lullabies
}

\author{
Patrick E. Savage ${ }^{1 *}$, Yuto Ozaki ${ }^{1}$, Sandra E. Trehub ${ }^{2}$ \\ ${ }^{1}$ Faculty of Environment and Information Studies, Keio University, Fujisawa, Japan \\ ${ }^{2}$ Department of Psychology, University of Toronto, Canada \\ *psavage@sfc.keio.ac.jp
}

\begin{abstract}
Please note: This is a non-peer-reviewed preprint. We welcome questions, comments, citation, and constructive criticism, bearing in mind that this is a non-peer-reviewed draft subject to revision. Please direct correspondence to psavage@sfc.keio.ac.jp.

Recommended citation: Savage, P. E., Ozaki, Y., \& Trehub, S. E. (2021). Sampling criteria and recording quality influence relaxation responses to lullabies. PsyArXiv preprint. https://doi.org/10.31234/osf.io/dcg7m
\end{abstract}

\section{ARISING FROM C. M. Bainbridge, M. Bertolo, et al. Nature Human Behaviour https://doi.org/10.1038/s41562-020-00963-z (2021)}

Short abstract: The original paper's sampling criteria involved selecting lullabies that adults rated as most likely to soothe a baby and non-lullabies rated as least likely to do so. Our analysis shows that lullabies in the stimulus set had systematically higher recording quality than non-lullabies, and those differences in recording quality were substantially greater than the paper's primary pre-registered analysis comparing infant heart rate when listening to lullabies vs. non-lullabies (original effect size: $d=0.23$ ). Accordingly, the authors' conclusion that infants relax more in response to unfamiliar foreign lullabies than to non-lullabies may be an artefact of their sampling methods.

Bainbridge, Bertolo, et al. ${ }^{1}$ report that a large sample of US infants relax in response to eight unfamiliar foreign lullabies in comparison to eight non-lullaby controls (love songs, healing songs, or dance songs) matched for vocalist gender, number of singers, and instrumentation. The authors found a significant effect on their pre-registered outcome variable of heart rate with this sample of 16 songs. However, their sampling criteria for the 16 songs call into question the generality of their claim that these effects are specific to lullabies.

The main issue is that the eight lullabies and eight non-lullabies were neither matched nor randomly sampled with respect to the crucial variable of "soothingness" - instead, the samples were as unmatched as possible on this crucial variable:

"using adults' ratings of the songs from a previous study, we chose a set of lullabies rated as likely to be 'used to soothe a baby' and a set of non-lullabies with low ratings on that item.... We ordered the pairs such that those with larger differences on the rating 'used to soothe a baby' were presented first, so as to maximize the measurable differences in responses to lullabies versus non-lullabies in each infant, even if they became inattentive or fussy partway through the study." 
These sampling criteria introduced a bias favouring the predicted soothing response. Western listeners sometimes misidentify foreign non-lullabies as lullabies if they contain soothing acoustic features (e.g., slow tempo, low pitch $)^{2-4}$, but such soothing non-lullabies were systematically excluded from the sample, along with non-soothing lullabies. If the sampling criteria were reversed to include the non-lullabies rated as most likely to be used for soothing a baby and the lullabies least likely to be soothe, the finding would likely be reversed such that infants relaxed in response to non-lullabies. Accordingly, the title claim about an effect specific to lullabies is not supported by the data - instead, "lullabies" in the title should be replaced with "soothing songs" since the key variable manipulated was soothingness, rather than (or in addition to) song function.

Even with their sampling criteria, the effect size of the main pre-registered analysis of $d=0.23^{1}$ was substantially weaker than the cutoff of $\mathrm{d}=0.4$ suggested as a "smallest effect size of interest" 5 , and the effect lasted for a mere 5 seconds (from approximately 3-8 seconds into the 14-second trial; cf. Fig. $2 b$ in the paper). We predict that this finding would replicate with these 8 pairs of songs or with a sample of soothing and non-soothing songs irrespective of lullaby status, but not necessarily with a sample of randomly selected lullabies and non-lullabies. Accordingly, the reported result may be an example of replicable effects with limited interpretability ${ }^{6}$ or generalizability ${ }^{7}$.

Listening to the stimuli provided by the authors (https://osf.io/2t6cy/), we were struck by the high levels of noise in some of the non-lullaby examples despite the authors' statement that recordings were "manually edited to remove background noise and microphone artefacts". All 8 of the lullabies were recorded after 1947, but 3 of the 8 non-lullabies were recorded before 1947 using rudimentary recording technology, with substantial noise remaining even after manual editing. For example, in the matched pair of stimuli from 2:30-3:00 at https://osf.io/7ekfg/, the median subjective rating of audio quality (8-point scale) by 10 experts for the first recording of a Chuuk love song recorded in 1907 was 1 ("terrible quality"), while the second recording of an Ona lullaby recorded in 1966 was much higher (4: "sort of poor quality").

Analysis of the recording quality of the 8 lullabies vs. the 8 matched non-lullabies confirmed that the non-lullabies had systematically poorer average recording quality (Fig. 1). The effect size of this difference was substantially larger than the original paper's main pre-registered effect size $(\mathrm{d}$ $=0.23)$, whether measured through automated analysis of signal-to-noise ratio $(\mathrm{d}=0.99)$ or through subjective expert ratings $\left(\mathrm{d}=0.84^{2}\right.$; see Supplementary Materials for detailed methods). Thus, the reported differences in soothing effects may be attributable to recording quality and other acoustic features known to influence soothing quality such as tempo or pitch height ${ }^{3}$ rather than features unique to lullabies. Note that recording quality was fairly low in general, with mean

\footnotetext{
${ }^{1}$ Note that the paper reports instead a larger effect size of $d=0.36$, but this was comparing lullabies vs. baseline, rather than the main pre-registered analysis comparing lullabies vs. non-lullabies. We calculated the pre-registered effect size of $d=0.23$ by adding the following script to the code provided by the authors at https://github.com/themusiclab/infant-relax: cohensD( $x=h r$ joined\$smean_lul_hr, $y=h r \_j o i n e d \$$ mean_nlul_hr, method = "paired")

${ }^{2}$ Full statistics: signal-to-noise ratio $\mathrm{d}=0.99 ; \mathrm{t}=2.8, \mathrm{df}=7, \mathrm{p}=0.03$; subjective ratings $\mathrm{d}=0.84, \mathrm{t}=2.7, \mathrm{df}=9, \mathrm{p}$ $=0.02$; original pre-registered comparison of heart rate in lullabies vs. non-lullabies $\mathrm{d}=0.23 ; \mathrm{t}=2.75, \mathrm{df}=138, \mathrm{p}=$ 0.007).
} 
ratings for lullabies and non-lullabies falling between 4 ("sort of poor quality") and 5 ("sort of good quality").
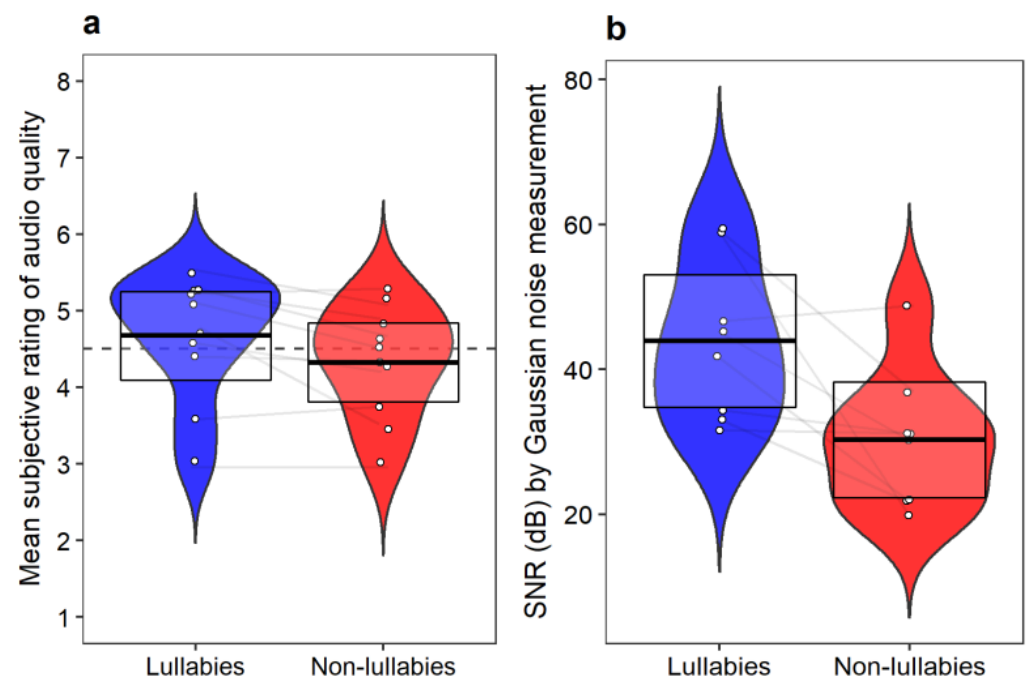

Figure 1. The 8 lullabies chosen as experimental stimuli had systematically higher recording quality than the 8 paired non-lullabies, whether audio quality was measured using a) subjective ratings, or b) automated acoustic analysis. a) Each data point represents ratings by experts on a scale of 1 ("terrible quality") - 8 ("perfect quality") averaged across the 8 lullabies (left) or 8 non-lullabies (right; $n=10$ raters). b) Each data point represents the signal-to-noise ratio calculated for each of the 8 lullabies (left) or 8 non-lullabies (right; $n=8$ pairs of audio recordings). Gray lines indicate the pairs of points that represent the same rater (a) or same pair of song stimuli (b); the violin plots (coloured areas) are kernel density estimations; the horizontal black lines indicate means, and the shaded white boxes indicate the $95 \%$ confidence intervals of the means. The violin plots use code adapted from Fig. 2 in the original paper.

The finding that US infants are soothed by foreign songs rated as likely to soothe an infant may be consistent with the proposed evolutionary theory focused on the adaptive function of lullabies ${ }^{8}$, but it is also consistent with alternative hypotheses highlighting the social powers of music regardless of genre ${ }^{9,10}$ or with almost any other hypothesis, even those arguing that music is an evolutionarily useless byproduct of language evolution ${ }^{11}$. Future studies with cross-cultural music samples either unselected for soothingness ${ }^{12}$ or matched on that quality would reveal the generalizability of the findings and their implications for theories of the evolution of musicality.

\section{Data availability:}

Data and source code for the new analyses presented are available at https://github.com/compmusic-lab/lullaby-analysis.

\section{Acknowledgments:}

We thank the authors for publishing detailed data, code, stimuli, and pre-registration documents that allowed us to better understand their methodology. We thank E. Benetos, H. Fukatsu, H. Kondo, S. Mani, J. McBride, P. Pfordresher, P. Protskouva, A. Ragano, E. Sakai, and J. Six for 
their ratings of audio quality. We thank E. Benetos, M. Bertolo, H. Daikoku, J. McBride, A. Ragano, and S. Passmore for feedback on earlier versions of this manuscript.

\section{Author contributions:}

P.E.S., S.E.T, and Y.O. designed the analyses; Y.O. and P.E.S. performed the analyses; P.E.S. drafted the main text, Y.O. drafted the supplementary material. All authors contributed additional editing and approved the manuscript.

\section{Competing interests:}

None.

\section{References:}

1. Bainbridge, C. M., Bertolo, M., et al. Infants relax in response to unfamiliar foreign lullabies. Nat. Hum. Behav. 5, 256-264 (2021).

2. Trehub, S. E., Unyk, A. M. \& Trainor, L. J. Adults identify infant-directed music across cultures. Infant Behav. Dev. 16, 193-211 (1993).

3. Cirelli, L. K., Jurewicz, Z. B. \& Trehub, S. E. Effects of maternal singing style on motherinfant arousal and behavior. J. Cogn. Neurosci. 32, 1213-1220 (2020).

4. Mehr, S. A., Singh, M., York, H., Glowacki, L. \& Krasnow, M. M. Form and function in human song. Curr. Biol. 28, 356-368 (2018).

5. Lakens, D. Equivalence tests: A practical primer for t tests, correlations, and metaanalyses. Soc. Psychol. Personal. Sci. 8, 355-362 (2017).

6. Mehr, S. A., Scannell, D. A. \& Winner, E. Sight-over-sound judgments of music performances are replicable effects with limited interpretability. PLoS One 13, e0202075 (2018).

7. Yarkoni, T. The generalizability crisis. Behav. Brain Sci. 1-37 (2020). doi:10.1017/S0140525X20001685

8. Mehr, S. A., Krasnow, M. M., Bryant, G. A. \& Hagen, E. H. Origins of music in credible signaling. Behav. Brain Sci. 23-39 (2021). doi:10.1017/S0140525X20000345

9. Savage, P. E. et al. Music as a coevolved system for social bonding. Behav. Brain Sci. 122 (2021). doi:10.1017/S0140525X20000333

10. Trehub, S. E., Becker, J. \& Morley, I. Cross-cultural perspectives on music and musicality. in The origins of musicality (ed. Honing, H.) 129-148 (MIT Press, 2018).

11. Pinker, S. How the mind works. (Norton, 1997).

12. Savage, P. E. An overview of cross-cultural music corpus studies. in Oxford Handbook of Music and Corpus Studies (eds. Shanahan, D., Burgoyne, A. \& Quinn, I.) (Oxford University Press). doi:10.31235/osf.io/nxtbg 
Supplementary Information:

\section{$\underline{\text { Summary of the audio quality evaluations }}$}

We quantified the audio quality of the 16 stimuli in two different ways: 1) subjective ratings, and 2 ) automated analysis. For both methods, we extracted the 14-second stimuli directly from the from the video stimuli IPL01.mp4 (https://osf.io/f46wj/) and IPL02.mp4 (https://osf.io/nhp26/). Comparing these audio files with the original audio files at https://osf.io/vcybz showed that these stimuli had been partially de-noised from the originals, but not completely.

\section{Subjective ratings}

We collected subjective ratings of audio quality from 10 experts using an 8-point scale adapted from the scale used by Mehr et al. ${ }^{13}$ to rate transcription quality, as follows:

Audio recording quality (this encompasses things like noise, clipping, harshness, muffled sounds, etc.):

1: Terrible quality

2: Extremely poor quality

3: Very poor quality

4: Sort of poor quality

5: Sort of good quality

6: Very good quality

7: Extremely good quality

8: Perfect quality

Raters were asked only to rate the audio quality, and were blind to both our hypotheses and to song metadata (i.e., they were not aware of song function, society of origin, etc., nor were they aware that our goal was to compare audio quality with respect to song function). We invited these experts because they had experience listening to ethnographic recordings of diverse songs as part of our ongoing research ${ }^{14}$. Mean ratings by each rater collapsed across lullabies and across non-lullabies are plotted in Figure 1.

\section{$\underline{\text { Automated analysis (signal-to-noise ratio) }}$}

\section{Summary}

The quality of the audio embedded in the video stimulus is evaluated by the signal-to-noise ratio. Although Bainbridge et al. manually manipulated original audio data of the Natural History of Song dataset to remove noise, the degree of noise remaining was unequally balanced between lullaby song data and non-lullaby song data. Hence, the discrepancy of noise level between each pair of songs is investigated. The audio quality is quantified by the signal-to-noise ratio (SNR), with the 16 audio files extracted directly from the video stimuli IPL01.mp4 (https://osf.io/f46wj/) and IPL02.mp4 (https://osf.io/nhp26/).

\section{Methods}


The SNR evaluates the ratio of the power of the signal and noise as follows. SNR takes the noise as the denominator. Hence, the lesser noise, the higher SNR, and the better audio quality.

$$
\begin{aligned}
\mathrm{SNR} & =\frac{P_{\text {signal }}}{P_{\text {noise }}} \\
\mathrm{SNR}(\mathrm{dB}) & =10 \log _{10} \frac{P_{\text {signal }}}{P_{\text {noise }}}
\end{aligned}
$$

Basically, SNR is evaluated by an original clean signal and noise, which is called a priori SNR. However, we neither have a clean signal of audio nor the noise-only signal in our case which means we cannot use established audio quality evaluation protocols such as PEAQ ${ }^{15}$. So we need to estimate the signal power of clean audio and noise from the actual audio already containing noise. There is a standardized way to evaluate the SNR using only noisy signals (i.e. single-ended evaluation) such as P.563 ITU-T Standard established in the field of telephony technology ${ }^{16}$, but this scheme requires audio to have a certain length of noise-only segment (e.g. unvoiced segment of speech) to estimate the noise. Our audio files do not meet this requirement, so the method proposed by Gerkmann \& Hendriks ${ }^{17}$ is chosen to estimate the degree of noise. The sampling frequency of audio is $48 \mathrm{kHz}$, and the STFT segment length is 8192 points with $75 \%$ overlap using the flap-top window $\left(\right.$ HFT90D $\left.^{18}\right)$ to obtain better amplitude accuracy. The parameters we used are: 0.95 for $\alpha$ of recursive smoothing of the estimated noise variance and 15 for $\xi$ of a priori SNR dB. Although the chosen method has parameters to prevent stagnation of the noise power update due to an underestimation of the noise power, we did not use these parameters since we did not observe such an issue when experimenting with synthesized audio and an external vocal song dataset ${ }^{19}$. We also confirmed this setting could produce reasonable estimation according to the simulation with the external dataset written below. We used the median of the latter half of the estimation as the noise power. Figures S1 below shows a sample of our estimation. Originally, the chosen method is proposed for speech, but the key technical assumptions of the methods are that noise is uncorrelated with the signal and both the power of clean signal and noise follows Gaussian distributions, and we consider this applicable to monophonic singing audio data.

However, our approach has two limitations. One is that our estimation targets Gaussian noise, but SNR measured by Gaussian noise does not necessarily correlate to human perception of audio quality. The second issue is that some sounds inherent in human vocals (e.g. breathing) are also regarded as Gaussian noise, and the amount of such voice-caused noise limits the attainable minimum estimation of noise power. This is exemplified by figure $\mathrm{S} 2$. The horizontal lines are measured SNR from the original clean audio, and scatter plots take the y-axis as estimated SNR and the $\mathrm{X}$-axis as the ground-truth SNR that noise is randomly generated. Ideally, the horizontal lines should be positioned at infinity because it is calculated from noise-free signals, but this demonstrates our SNR is not purely the ratio of human vocal vs. other sounds. Although the amount of voice-caused noise depends on the way of singing, it shows that noise power smaller than voice-caused noise cannot be estimated. Meanwhile, we can also see the chosen method can properly detect the power of Gaussian noise from monophonic singing recordings.

Even though the limitations described above exist, our approach can estimate the amount of Gaussian noise mixed in human vocal recordings, and our analysis confirms that noise power is not equally distributed among song stimuli in terms of simple Gaussian noise. Applying this 
method of SNR estimation to the 8 lullaby-non lullaby pairs of song stimuli revealed that the nonlullabies had systematically lower SNR than the lullabies (Fig. 1).

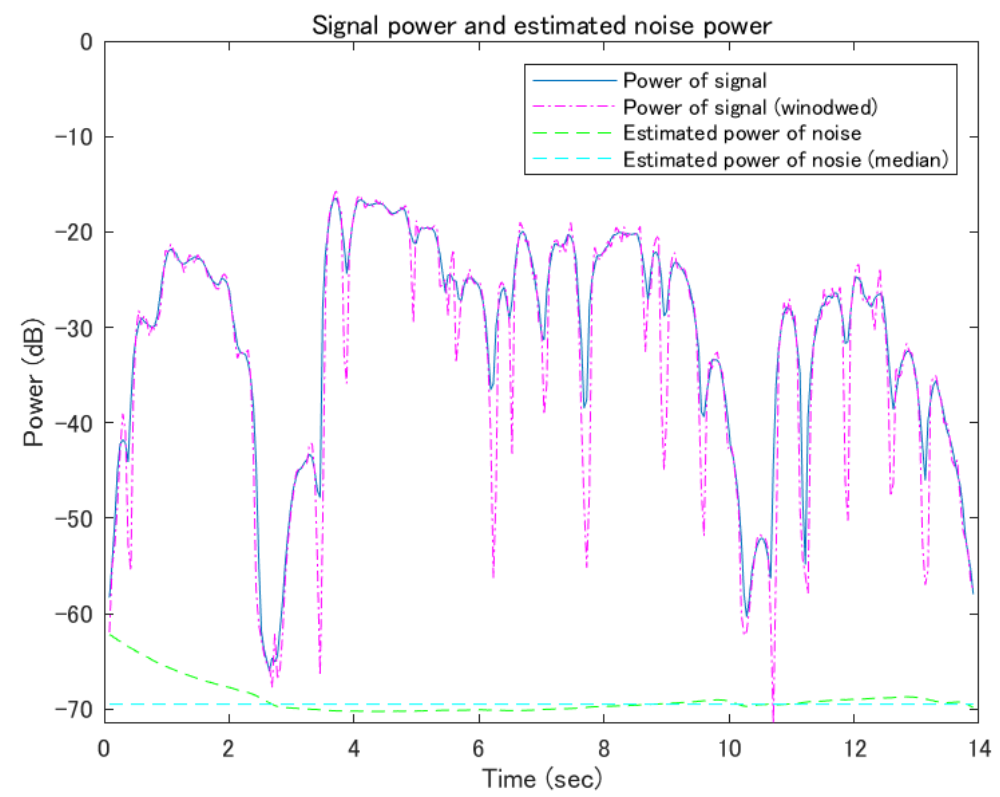

Figure S1: Noise power estimate of NAIV-043 for example.

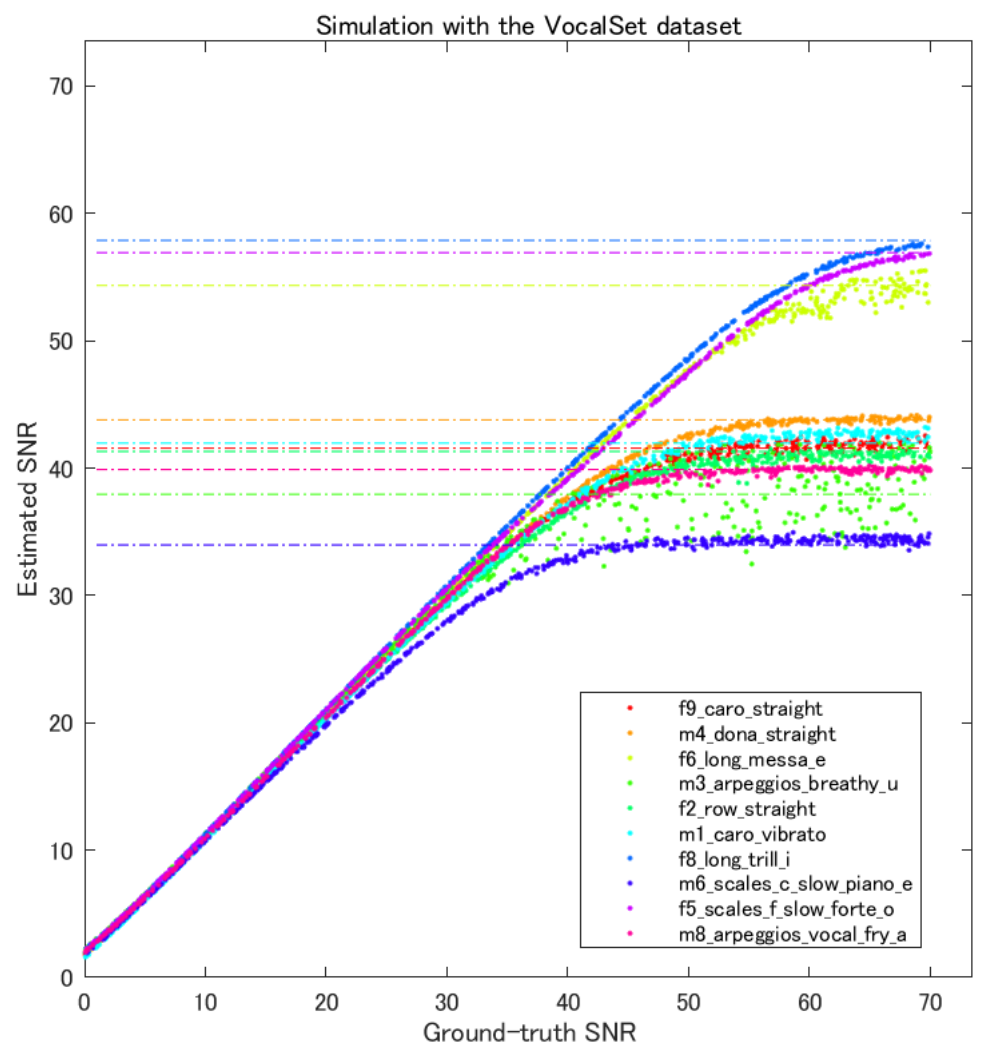


Figure S2: Simulation of noise estimation with the subset songs of VocalSet dataset.

\section{Supplementary References}

13. Mehr, S. A. et al. Universality and diversity in human song. Science 366, eaax0868 (2019).

14. Ozaki, Y. et al. Agreement among human and automated transcriptions of global songs. in Proceedings of the 22nd International Conference on Music Information Retrieval (ISMIR) (2021) doi:10.31234/osf.io/jsa4u

15. Thiede, T. et al. PEAQ - The ITU Standard for Objective Measurement of Perceived Audio Quality. J. Audio Eng. Soc. 48, 3-29 (2000).

16. Malfait, L., Berger, J. \& Kastner, M. P.563 - The ITU-T Standard for Single-Ended Speech Quality Assessment. IEEE Trans. Audio. Speech. Lang. Process. 14, 1924-1934 (2006).

17. Gerkmann, T. \& Hendriks, R. C. Unbiased MMSE-Based Noise Power Estimation with Low Complexity and Low Tracking Delay. IEEE Trans. Audio. Speech. Lang. Process. 20, 1383-1393 (2012).

18. Heinzel, G., Rüdiger, A. \& Schilling, R. Spectrum and spectral density estimation by the Discrete Fourier transform (DFT), including a comprehensive list of window functions and some new flat-top windows. Max Planck Inst. Gravitational Phys. 1-84 (2002).

19. Wilkins, J., Seetharaman, P., Wahl, A. \& Pardo, B. Vocalset: A singing voice dataset. Proc. 19th Int. Soc. Music Inf. Retr. Conf. 468-474 (2018). 